\title{
On Fourier Series of Jacobi-Sobolev Orthogonal Polynomials
}

\author{
F. MARCELLÁN ${ }^{1}$, B. P. OSILENKER ${ }^{2}$, and I. A. ROCHA ${ }^{3}$
}

${ }^{1}$ Dpto. de Matemáticas, Universidad Carlos III de Madrid, Avda. Universidad 20, 28911 Leganés, Madrid (SPAIN) < pacomarc@ing.uc3m.es> ${ }^{2}$ Dep. of Math., Moscow State Civil Engineering University, Moscow (RUSSIA)

$<$ b_osilenker@mail.ru> ; ${ }^{3}$ Dpto.de Matemática Aplicada, E.U.I.T.

Telecomunicación, Universidad Politécnica de Madrid, Ctra. de Valencia Km.

7, 28031 Madrid (SPAIN) <igalvar@euitt.upm.es >

(Received on October 16 2000; Revised February 26 2001)

Let $\mu$ be the Jacobi measure on the interval $[-1,1]$ and introduce the discrete Sobolev-type inner product

$$
\langle f, g\rangle=\int_{-1}^{1} f(x) g(x) d \mu(x)+M f(c) g(c)+N f^{\prime}(c) g^{\prime}(c)
$$

where $c \in(1, \infty)$ and $M, N$ are non negative constants such that $M+N>0$. The main purpose of this paper is to study the behaviour of the Fourier series in terms of the polynomials associated to the Sobolev inner product. For an appropriate function $f$, we prove here that the Fourier-Sobolev series converges to $f$ on the interval $(-1,1)$ as well as to $f(c)$ and the derivative of the series converges to $f^{\prime}(c)$. The term appropriate means here, in gencral, the same as we need for a function $f(x)$ in order to have convergence for the series of $f(x)$ associated to the standard inner product given by the measure $\mu$. No additional conditions are needed.

Keywords: Orthogonal polynomials; Sobolev inner product; Fourier series.

Classification: AMS Subject Classification: 42C05

ISSN 1025-5834 print; ISSN 1029-242X. (C) 2002 Taylor \& Francis Ltd DOI: $10.1080 / 1025583021000022450$ 


\section{INTRODUCTION}

Let $\mu$ be a finite positive Borel measure on the interval $[-1,1]$ such that supp $\mu$ is an infinite set and let $c$ be a real number on $(1, \infty)$. For $f$ and $g$ in $L^{2}(\mu)$ such that there exits the first derivative in $c$ we can introduce the Sobolev-type inner product

$$
\langle f, g\rangle=\int_{-1}^{1} f(x) g(x) d \mu(x)+M f(c) g(c)+N f^{\prime}(c) g^{\prime}(c)
$$

where $M$ and $N$ are non-negative real numbers with $M+N>0$. Let $\left(\hat{B}_{k}(x)\right)_{k=0}^{\infty}$ the sequence of orthonormal polynomials with respect to this inner product

$$
\left\langle\hat{B}_{n}(x), \hat{B}_{k}(x)\right\rangle=\delta_{n, k} \quad k, n=0,1, \ldots
$$

For every function $f$ such that $\left\langle f, \hat{B}_{k}\right\rangle$ exists for $k=0,1, \ldots$ we introduce the associated Fourier-Sobolev series

$$
\sum_{k=0}^{\infty}\left\langle f, \hat{B}_{k}\right\rangle \hat{B}_{k}(x)
$$

The main purpose of this paper is the proof of the relation

$$
\sum_{k=0}^{\infty}\left\langle f, \hat{B}_{k}\right\rangle \hat{B}_{k}(x)=f(x), \quad x \in(-1,1) \cup\{c\}, \quad \sum_{k=0}^{\infty}\left\langle f, \hat{B}_{k}\right\rangle \hat{B}_{k}^{\prime}(c)=f^{\prime}(c)
$$

for the Jacobi measure $d \mu(x)=(1-x)^{\alpha}(1+x)^{\beta} d x, \alpha>-1, \beta>-1$, under standard sufficient conditions for $f$. The precise terms of this result are given in Section 4.

In order to obtain this result we need previously some estimates for $\hat{B}_{k}(x)$ in $[-1,1] \cup\{c\}$ and also for $\hat{B}_{k}^{\prime}(c)$. They are obtained in Section 3 not only for the Jacobi measure but for any measure $\mu$ which belongs to Szegö class. We start with a representation of $\hat{B}_{k}(x)$ in terms of the polynomials $\left(q_{n}(x)\right)_{n=0}^{\infty}$ orthonormal with respect to the measure $(c-x)^{2} d \mu(x)$. In Section 2 we prove that

$$
\hat{B}_{n}(x)=A_{n} q_{n}(x)+B_{n} q_{n-1}(x)+C_{n} q_{n-2}(x)
$$


and that the constants $A_{n}, B_{n}, C_{n}$ have limit points provided that the measure $\mu$ has ratio asymptotics. One consequence of this result is the asymptotics for the polynomials $\hat{B}_{k}(x)$ and the asymptotic behaviour of their zeros. This is well known from papers by G. López, F. Marcellán and W. Van Assche ([2], [4]) where they solved this problem using a different representation of the polynomials $\hat{B}_{k}(x)$.

The fact that the point $c$ is outside the interval $[-1,1]$ plays an important role in the whole paper because it allows the function $1 /(x-c)^{2}$ to be continuous in the interval and the Sobolev space behaves as a vector space with two real components and the other on $L^{2}(\mu)$. Notice that some estimates of polynomials $\hat{B}_{k}$ when $c=1$ have been obtained in [1]. It remains open the problem of the estimates when $c \in(-1,1)$.

\section{ASYMPTOTIC FORMULAS}

We will denote by $\left(p_{n}(x)\right)_{n=0}^{\infty}$ the sequence of orthonormal polynomials with respect to $d \mu(x)$ and by $\left(\tilde{q}_{n}(x)\right)_{n=0}^{\infty}$ and $\left(q_{n}(x)\right)_{n=0}^{\infty}$ the orthonormal sequences with respect to $(c-x) d \mu(x)$ and $(c-x)^{2} d \mu(x)$ respectively. We will also denote by $k\left(\pi_{n}\right)$ the leading coefficient of any polynomial $\pi_{n}(x)$ and $\hat{B}_{n}(x)$ the orthonormal polynomials with respect to the inner product (1) as it was said.

Since there are important differences for the different choices of $M$ and $N$, we will start with $M>0$ and $N>0$ and, in the next subsection, cases $N=0$ and $M=0$ will be studied separately.

\subsection{Case $M>0, N>0$}

In this paragraph, we assume that $\mu^{\prime}>0$ a.e., i.e., the polynomials $P_{n}(x), \tilde{q}_{n}(x)$ and $q_{n}(x)$ have ratio asymptotics.

THEOREM 2.1 If $M>0$ and $N>0$, there are real constants $A_{n}, B_{n}$ and $C_{n}$ such that

$$
\hat{B}_{n}(x)=A_{n} q_{n}(x)+B_{n} q_{n-1}(x)+C_{n} q_{n-2}(x), \quad n=0,1,2 \ldots
$$




\section{Moreover}

$$
\lim _{n \rightarrow \infty} A_{n}=\frac{c-\sqrt{c^{2}-1}}{2}, \quad \lim _{n \rightarrow \infty} B_{n}=-1, \quad \lim _{n \rightarrow \infty} C_{n}=\frac{c+\sqrt{c^{2}-1}}{2}
$$

Proof Since $\hat{B}_{n}(x)=\sum_{j=0}^{n} a_{n, j} q_{j}(x)$ and

$$
\begin{aligned}
a_{n, j} & =\int_{-1}^{1} \hat{B}_{n}(x) q_{j}(x)(x-c)^{2} d \mu(x) \\
& =\left\langle\hat{B}_{n}(x),(x-c)^{2} q_{j}(x)\right\rangle=0, \quad j=0,1, \ldots n-3,
\end{aligned}
$$

then

$$
\begin{aligned}
\hat{B}_{n}(x) & =a_{n}, n q_{n}(x)+a_{n, n-1} q_{n-1}(x)+a_{n}, n-2 q_{n-2}(x) \\
& =A_{n} q_{n}(x)+B_{n} q_{n-1}(x)+C_{n} q_{n-2}(x) .
\end{aligned}
$$

On the other hand, since

$$
\begin{aligned}
A_{n}^{2}+B_{n}^{2}+C_{n}^{2} & =\int_{-1}^{1} \hat{B}_{n}^{2}(x)(x-c)^{2} d \mu(x) \\
& \leq(c+1)^{2} \int_{-1}^{1} \hat{B}_{n}^{2}(x) d \mu(x) \leq(C+1)^{2},
\end{aligned}
$$

the coefficients $A_{n}, B_{n}$ and $C_{n}$ are bounded.

Denoting by $k\left(q_{n}\right)$ and $k\left(\hat{B}_{n}\right)$ the leading coefficients of $q_{n}(x)$ and $\hat{B}_{n}(x)$ respectively, we get

$$
A_{n}=\frac{k\left(\hat{B}_{n}\right)}{k\left(q_{n}\right)},
$$

and

$$
\begin{aligned}
C_{n} & =\int_{-1}^{1} \hat{B}_{n}(x) q_{n-2}(x)(x-c)^{2} d \mu(x)=\left\langle\hat{B}_{n}(x),(x-c)^{2} q_{n-2}(x)\right\rangle \\
& =\frac{k\left(q_{n-2}\right)}{k\left(\hat{B}_{n}\right)}=\frac{k\left(q_{n-2}\right)}{k\left(q_{n}\right)} \frac{1}{A_{n}} .
\end{aligned}
$$


Because $1 / x-c$ and $1 /(x-c)^{2}$ are continuous functions on $[-1,1]$ and, in particular, they belong to $L^{2}\left((x-c)^{2} d \mu(x)\right)$,

$$
\lim _{n \rightarrow \infty} \int_{-1}^{1} q_{n}(x) d \mu(x)=\lim _{n \rightarrow \infty} \int_{-1}^{1} \frac{q_{n}(x)}{(x-c)^{2}}(x-c)^{2} d \mu(x)=0
$$

and

$$
\lim _{n \rightarrow \infty} \int_{-1}^{1} q_{n}(x)(x-c) d \mu(x)=\lim _{n \rightarrow \infty} \int_{-1}^{1} \frac{q_{n}(x)}{x-c}(x-c)^{2} d \mu(x)=0 .
$$

Then

$$
\begin{aligned}
\lim _{n \rightarrow \infty} \int_{-1}^{1} q_{n}(x) \hat{B}_{n}(x) d \mu(x)= & \lim _{n \rightarrow \infty} \int_{-1}^{1} q_{n}(x)\left[\hat{B}_{n}(c)\right. \\
& \left.+\hat{B}_{n}^{\prime}(c)(x-c)\right] d \mu(x)=0 \\
\lim _{n \rightarrow \infty} \int_{-1}^{1} q_{n}(x) \hat{B}_{n+1}(x) d \mu(x)= & \lim _{n \rightarrow \infty} \int_{-1}^{1} q_{n}(x)\left[\hat{B}_{n+1}(c)\right. \\
& \left.+\hat{B}_{n+1}^{\prime}(c)(x-c)\right] d \mu(x)=0
\end{aligned}
$$

because $\hat{B}_{n}(c)$ and $\hat{B}_{n}^{\prime}(c)$ are bounded from the orthonormality of $\hat{B}_{n}(x)$.

Let $\Lambda$ be a family of non negative integers such that $\lim _{n \in \Lambda} A_{n}=a$ and $\lim _{n \in \Lambda} B_{n}=b$. As it is well known, (see [5] and [6]), if $\mu^{\prime}>0$ a.e. then $\lim _{n \in \Lambda} C_{n}=1 / 4 a$ (notice that $a>0$ because $C_{n}$ are bounded) and

$$
\lim _{n \rightarrow \infty} \int_{-1}^{1} \frac{q_{n}(x) q_{n+k}(x)}{(x-c)^{2}}(x-c)^{2} d \mu(x)=\frac{1}{\pi} \int_{-1}^{1} \frac{T_{k}(x)}{(x-c)^{2}} \frac{d x}{\sqrt{1-x^{2}}}
$$

where $T_{k}(x)=\cos (k \theta), x=\cos \theta$, are the Tchebichef polynomials of the first kind. 
As a consequence, from (2) and (3) and Theorem 2.1 we obtain $0=\lim _{n \in \Lambda} \int_{-1}^{1} q_{n}(x) \hat{B}_{n}(x) d \mu(x)=\frac{1}{\pi} \int_{-1}^{1} \frac{a+b T_{1}(x)+1 / 4 a T_{2}(x)}{(x-c)^{2}} \frac{d x}{\sqrt{1-x^{2}}}$,

$$
\begin{aligned}
0 & =\lim _{n \in \Lambda} \int_{-1}^{1} q_{n}(x) \hat{B}_{n+1}(x) d \mu(x) \\
& =\frac{1}{\pi} \int_{-1}^{1} \frac{a T_{1}(x)+b+1 / 4 a T_{1}(x)}{(x-c)^{2}} \frac{d x}{\sqrt{1-x^{2}}}
\end{aligned}
$$

Denoting

$$
\begin{gathered}
\Pi_{1}(x)=\left(4 a^{2}+1\right) T_{1}(x)+4 a b=\left(4 a^{2}+1\right) x+4 a b, \\
\Pi_{2}(x)=T_{2}(x)+4 a b T_{1}(x)+4 a^{2}=2 x^{2}+4 a b x+4 a^{2}-1,
\end{gathered}
$$

and

$$
\omega(z)=\frac{-1}{\sqrt{z^{2}-1}}=\frac{1}{\pi} \int_{-1}^{1} \frac{1}{x-z} \frac{d x}{\sqrt{1-x^{2}}},
$$

(5) becomes

$$
\begin{aligned}
0 & =\frac{1}{\pi} \int_{-1}^{1} \frac{\Pi_{1}(x)}{(x-c)^{2}} \frac{d x}{\sqrt{1-x^{2}}}=\frac{1}{\pi} \int_{-1}^{1} \frac{\Pi_{1}(c)+\Pi_{1}^{\prime}(c)(x-c)}{(x-c)^{2}} \frac{d x}{\sqrt{1-x^{2}}} \\
& =\left(\Pi_{1} \omega\right)^{\prime}(c)=\frac{4 a b c+4 a^{2}+1}{\left(c^{2}-1\right)^{3 / 2}}
\end{aligned}
$$

which means that $b=-(1 / c)(a+(1 / 4 a))$.

Analogously, (4) becomes

$$
0=\left(\Pi_{2} \omega\right)^{\prime}(c)+2
$$

and it gives

$$
4 a^{2}=\left(\varphi^{-}(c)\right)^{2}=:\left(c-\sqrt{c^{2}-1}\right)^{2}
$$


Since $a>0, a=\varphi^{-}(c) / 2$ and $b=-1$. As a consequence the only limit points of $A_{n}$ and $B_{n}$ are $\varphi^{-}(c) / 2$ and -1 respectively and the theorem is proved

As a straightforward consequence of Theorem 2.1 one obtains the strong (resp. ratio) asymptotics for the polynomials $\hat{B}_{n}(x)$ provided that $\mu$ belongs to Szegö (resp. Nevai) class. These results were obtained by G. López, F. Marcellán and W. Van Assche in [2] and [4] using a different representation for $\hat{B}_{n}(x)$.

COROLLARY 2.1 With the previous conditions we have i)

$$
\lim _{n \rightarrow \infty} \frac{\hat{B}_{n}(x)}{q_{n}(x)}=\frac{\varphi^{-}(c)}{2}\left(1-\frac{\varphi(c)}{\varphi(x)}\right)^{2}
$$

uniformly on compact sets of $C \backslash[-1,1]$ and $\varphi(x)=x+\sqrt{x^{2}-1}$.

ii) $n-2$ zeros of $\hat{B}_{n}(x)$ are in $[-1,1]$ and the other 2 zeros tend to $c$. iii)

$$
\lim _{n \rightarrow \infty} \frac{\hat{B}_{n+1}(x)}{\hat{B}_{n}(x)}=x+\sqrt{x^{2}-1}
$$

uniformly on compact sets of $C \backslash([-1,1] \cup\{c\})$.

iv) If $\int_{-1}^{1} \log \mu^{\prime}(x) d x / \sqrt{1-x^{2}}>-\infty$ then

$$
\lim _{n \rightarrow \infty} \frac{\hat{B}_{n}(x)}{\left(x+\sqrt{x^{2}-1}\right)^{n}}=\frac{\varphi^{-}(c)}{2}\left(1-\frac{\varphi(c)}{\varphi(x)}\right)^{2} S(x)
$$

uniformly on compact sets of $\mathbb{C} \backslash[-1,1]$, where $S(x)$ is the Szegö function of $(x-c)^{2} \mu^{\prime}(x)$ (see [8], Th. 12.1.2 as well as the definition in page 276)

Item ii) is a consequence of the fact that $\int_{-1}^{1} \hat{B}_{n}(x)(x-c)^{2} x^{k} d \mu(x)=0$ for $k=0,1, \ldots, n-3$ and the asymptotic formula i).

The Sobolev polynomials satisfy a five term recurrence relation and its coefficients behave as the ones of standard orthogonal polynomials. 
THEOREM 2.2 There are constants $\alpha_{n}, \beta_{n}, \gamma_{n}$ such that

$$
\begin{gathered}
(x-c)^{2} \hat{B}_{n}(x)=\alpha_{n} \hat{B}_{n+2}(x)+\beta_{n} \hat{B}_{n+1}(x)+\gamma_{n} \hat{B}_{n}(x)+\beta_{n-1} \hat{B}_{n-1}(x) \\
+\alpha_{n-2} \hat{B}_{n-2}(x), \quad n \geq 0 . \\
\alpha_{-1}=\alpha_{-2}=\beta_{-1}=0 .
\end{gathered}
$$

Morower; if $\mu^{\prime}>0$ a.e. then

$$
\lim _{n \rightarrow \infty} \alpha_{n}=\frac{1}{4}, \quad \lim _{n \rightarrow \infty} \beta_{n}=-c, \quad \lim _{n \rightarrow \infty} \gamma_{n}=c^{2}+\frac{1}{2} .
$$

Proof Recurrence relation is a straightforward consequence of the fact that

$$
\left\langle(x-c)^{2} f(x), g(x)\right\rangle=\left\langle f(x),(x-c)^{2} g(x)\right\rangle .
$$

For the asymptotic behaviour of the coefficients we have

$$
\alpha_{n}=\frac{k\left(\hat{B}_{n}\right)}{k\left(\hat{B}_{n+2}\right)}=\frac{k\left(\hat{B}_{n}\right)}{k\left(q_{n}\right)} \frac{k\left(q_{n}\right)}{k\left(q_{n+2}\right)} \frac{k\left(q_{n+2}\right)}{k\left(\hat{B}_{n+2}\right)}
$$

and, if $\mu^{\prime}>0$ a.e., $\lim _{n \rightarrow \infty} \alpha_{n}=\lim _{n \rightarrow \infty} k\left(q_{n}\right) / k\left(q_{n+2}\right)=\frac{1}{4}$.

$$
\gamma_{n}=\left\langle(x-c)^{2} \hat{B}_{n}(x), \hat{B}_{n}(x)\right\rangle=\int_{-1}^{1}(x-c)^{2} \hat{B}_{n}^{2}(x) d \mu(x)=A_{n}^{2}+B_{n}^{2}+C_{n}^{2}
$$

where $\hat{B}_{n}(x)=A_{n} q_{n}(x)+B_{n} q_{n-1}(x)+C_{n} q_{n-2}(x)$. Then

$$
\lim _{n \rightarrow \infty} \gamma_{n}=\left(\frac{\varphi^{-}(c)}{2}\right)^{2}+1+\left(\frac{\varphi(c)}{2}\right)^{2}=c^{2}+\frac{1}{2}
$$


ON FOURIER SERIES OF JACOBI-SOBOLEV ORTHOGONAL POLYNOMIAL 681

Finally, from

$$
\begin{aligned}
\beta_{n} & =\left\langle(x-c)^{2} \hat{B}_{n}(x), \hat{B}_{n+1}(x)\right\rangle \\
& =\int_{-1}^{1}(x-c)^{2} \hat{B}_{n}(x) \hat{B}_{n+1}(x) d \mu(x)=A_{n} B_{n+1}+B_{n} C_{n+1},
\end{aligned}
$$

we get

$$
\lim _{n \rightarrow \infty} \hat{B}_{n}=-\left(\frac{\varphi(c)}{2}+\frac{\varphi^{-}(c)}{2}\right)=-c .
$$

THEOREM 2.3 If $\mu^{\prime}>0$ a.e. on $[-1,1]$ then

$$
\lim _{n \rightarrow \infty} \int_{-1}^{1} f(x) \hat{B}_{n}(x) \hat{B}_{n+k}(x) d \mu(x)=\frac{1}{\pi} \int_{-1}^{1} f(x) T_{k}(x) \frac{d x}{\sqrt{1-x^{2}}}
$$

for any continuous function on $[-1,1]$.

Proof

$$
\begin{aligned}
\int_{-1}^{1} f(x) & \hat{B}_{n}(x) \hat{B}_{n+k}(x) d \mu(x) \\
= & \int_{-1}^{1} \frac{f(x)}{(x-c)^{2}}\left[A_{n} q_{n}(x)+B_{n} q_{n-1}(x)+C_{n} q_{n-2}(x)\right] . \\
& \cdot\left[A_{n+k} q_{n+k}(x)+B_{n+k} q_{n+k-1}(x)+C_{n+k} q_{n+k-2}(x)\right](x-c)^{2} d \mu(x) .
\end{aligned}
$$


From the properties of $q_{n}(x)$ and taking into account that $A_{n} \rightarrow \varphi^{-}(c) / 2, B_{n} \rightarrow-1, C_{n} \rightarrow \varphi(c) / 2$ we have for $k \geq 2$

$$
\begin{aligned}
\lim _{n \rightarrow \infty} \int_{-1}^{1} f(x) \hat{B}_{n}(x) \hat{B}_{n+k}(x) d \mu(x) \\
=\frac{1}{\pi} \int_{-1}^{1} \frac{f(x)}{(x-c)^{2}}\left[\left(c^{2}+\frac{1}{2}\right) T_{k}(x)\right. \\
\quad+\frac{1}{4}\left(T_{k+2}(x)+T_{k-2}(x)\right)-c\left(T_{k+1}(x) .\right. \\
\left.\left.\quad+T_{k-1}(x)\right)\right] \frac{d x}{\sqrt{1-x^{2}}} \\
=\frac{1}{\pi} \int_{-1}^{1} \frac{f(x)}{(x-c)^{2}}\left(x^{2}-2 c x+c^{2}\right) T_{k}(x) \frac{d x}{\sqrt{1-x^{2}}} .
\end{aligned}
$$

In the same way, this relation holds for $k=0$ and $k=1$.

\subsection{Cases $N=0$ and $M=0$}

Because our goal is to study Fourier series in Jacobi-Sobolev polynomials, from now on we will assume that the measure $\mu$ belongs to the Szegö class, i.e.,

$$
\int_{-1}^{1} \log \mu^{\prime}(x) \frac{d x}{\sqrt{1-x^{2}}}>-\infty
$$

In case $N=0$ this is not important because the same proof given in Theorem 2.1 works here. However, in case $M=0$, the proof given here needs strong asymptotics for the polynomials $q_{n}(x)$.

THEOREM 2.4 i) If we assume $N=0$ in the inner product (1), then there are real constants $A_{n}$ and $B_{n}$ such that

$$
\hat{B}_{n}(x)=A_{n} \tilde{q}_{n}(x)+B_{n} \tilde{q}_{n-1}(x) \quad n=0,1 \ldots
$$


where $\tilde{q}_{n}(x)$ are the orthonormal polynomials with respect to $(c-x) d \mu(x)$. Moreover

$$
\lim _{n \rightarrow \infty} A_{n}=\left(\frac{c-\sqrt{c^{2}-1}}{2}\right)^{1 / 2}, \quad \lim _{n \rightarrow \infty} B_{n}=-\left(\frac{c+\sqrt{c^{2}-1}}{2}\right)^{1 / 2}
$$

ii) When $M=0$ in the inner product (1), there are constants $A_{n}, B_{n}$ and $C_{n}$ such that

$$
\hat{B}_{n}(x)=A_{n} q_{n}(x)+B_{n} q_{n-1}(x)+C_{n} q_{n-2}(x), \quad n=0,1, \ldots
$$

where $q_{n}(x)$ are the orthonormal polynomials with respect to $(c-x)^{2} d \mu(x)$. Moreover

$$
\lim _{n \rightarrow \infty} A_{n}=\lim _{n \rightarrow \infty} C_{n}=\frac{1}{2}, \quad \lim _{n \rightarrow \infty} B_{n}=-c
$$

Proof

i) Since

$$
\begin{aligned}
\int_{-1}^{1} \hat{B}_{n}(x) \tilde{q}_{j}(x)(c-x) d \mu(x) & =\left\langle\hat{B}_{n}(x),(c-x) \tilde{q}_{j}(x)\right\rangle \\
& =0, j=0,1, \ldots, n-2
\end{aligned}
$$

we have

$$
\hat{B}_{n}(x)=A_{n} \tilde{q}_{n}(x)+B_{n} \tilde{q}_{n-1}(x)
$$

Since $\hat{B}_{n}(c)$ are bounded because of the orthonormality condition, and $\tilde{q}_{n}(c)$ behaves like $\left(c+\sqrt{c^{2}-1}\right)^{n}$,

$$
\lim _{n \rightarrow \infty} \frac{\hat{B}_{n}(c)}{\tilde{q}_{n-1}(c)}=\lim _{n \rightarrow \infty}\left(A_{n} \frac{\tilde{q}_{n}(c)}{\tilde{q}_{n-1}(c)}+B_{n}\right)=0
$$


Taking into account that $A_{n}=k\left(\hat{B}_{n}\right) / k\left(\tilde{q}_{n}\right), B_{n}=-k\left(\tilde{q}_{n-1}\right) / k\left(q_{n}\right) 1 / A_{n}$ as well as $A_{n}$ and $B_{n}$ are bounded, from (6) we deduce

$$
0=\lim _{n \in \Lambda} \frac{\hat{B}_{n}(c)}{\tilde{q}_{n-1}(c)}=A\left(c+\sqrt{c^{2}-1}\right)-\frac{1}{2 A}
$$

for a family of non-negative integers $\Lambda$, where $A$ is a limit point of $A_{n}$. Then $A^{2}=c-\sqrt{c^{2}-1} / 2$ and i) is proved.

In case ii) we have

$$
\begin{aligned}
\int_{-1}^{1} \hat{B}_{n}(x) q_{j}(x)(c-x)^{2} d \mu(x) & =\left\langle\hat{B}_{n}(x),(x-c)^{2} q_{j}(x)\right\rangle \\
& =0, j=0, \ldots, n-3
\end{aligned}
$$

This yields $\quad \hat{B}_{n}(x)=A_{n} q_{n}(x)+B_{n} q_{n-1}(x)+C_{n} q_{n-2}(x)$. Moreover $C_{n}=k\left(q_{n-2}\right) / k\left(q_{n}\right) 1 / A_{n}$. From the boundedness of $\hat{B}_{n}^{\prime}(c)$ and the asymptotic properties of $q_{n}(x)$ we have

$$
0=\lim _{n \rightarrow \infty} \frac{\hat{B}_{n}^{\prime}(c)}{q_{n-2}^{\prime}(c)}=\lim _{n \rightarrow \infty}\left(A_{n} \frac{q_{n}^{\prime}(c)}{q_{n-2}^{\prime}(c)}+B_{n} \frac{q_{n-1}^{\prime}(c)}{q_{n-2}^{\prime}(c)}+\frac{k\left(q_{n-2}\right)}{k\left(q_{n}\right)} \frac{1}{A_{n}}\right)
$$

Let $\Lambda$ be a sequence of non negative integers such that $\lim _{n \in \Lambda} A_{n}=A$ and $\lim _{n \in \Lambda} B_{n}=B$ which exist because $A_{n}, B_{n}$ and $C_{n}$ are bounded. Then

$$
0=A \varphi^{2}(c)+B \varphi(c)+\frac{1}{4 A}
$$

where $\varphi(c)=c+\sqrt{c^{2}-1}$.

On the other hand,

$$
\left|-N \hat{B}_{n}^{\prime}(c) q_{n-1}^{\prime}(c)\right|=\left|\int_{-1}^{1} \hat{B}_{n}(x) q_{n-1}(x) d \mu(x)\right| \leq \frac{1}{c-1}
$$


and hence $\left|\hat{B}_{n}^{\prime}(c)\right| \leq K / n(\varphi(c))^{n-1}$ for some constant $K$. As a consequence, $\lim _{n \rightarrow \infty} \hat{B}_{n}^{\prime}(c) q_{n-1}(c)=0$. Taking into account that

$\int_{-1}^{1} \hat{B}_{n}(x) q_{n-1}(x)(c-x) d \mu(x)=\left\langle\hat{B}_{n}(x),(c-x) q_{n-1}(x)\right\rangle+N \hat{B}_{n}^{\prime}(c) q_{n-1}(c)$,

the last relation yields

$$
\begin{aligned}
\lim _{n \in \Lambda} \int_{-1}^{1} \hat{B}_{n}(x) q_{n-1}(x)(c-x) d \mu(x) & =\lim _{n \in \Lambda}\left\langle\hat{B}_{n}(x),(c-x) q_{n-1}(x)\right\rangle \\
& =-\lim _{n \in \Lambda}\left\langle\hat{B}_{n}(x), x q_{n-1}(x)\right\rangle \\
& =-\lim _{n \in \Lambda} \frac{k\left(q_{n-1}\right)}{k\left(\hat{B}_{n}\right)}=-\lim _{n \in \Lambda} \frac{k\left(q_{n-1}\right)}{k\left(q_{n}\right)} \frac{1}{A_{n}} \\
& =-\frac{1}{2 A} .
\end{aligned}
$$

But

$\lim _{n \in \Lambda} \int_{-1}^{1} \hat{B}_{n}(x) q_{n-1}(x)(c-x) d \mu(x)=\frac{1}{\pi} \int_{-1}^{1} \frac{(A+1 / 4 A) T_{1}(x)+B}{c-x} \frac{d x}{\sqrt{1-x^{2}}}$,

and thus we have

$$
\frac{1}{\pi} \int_{-1}^{1} \frac{(A+1 / 4 A) T_{1}(x)+B}{c-x} \frac{d x}{\sqrt{1-x^{2}}}=-\frac{1}{2 A} .
$$

After some calculations, equations (7) and (8) give $A=\frac{1}{2}$ and $B=-c$.

When the measure belongs to the Szegö class we have the following consequences

COROLlary 2.2 For $N=0$,

i)

$$
\lim _{n \rightarrow \infty} \frac{\hat{B}_{n}(x)}{\tilde{q}_{n}(x)}=\left(\frac{\varphi^{-}(c)}{2}\right)^{1 / 2}\left(1-\frac{\varphi(c)}{\varphi(x)}\right)
$$


uniformly on compact sets of $C \backslash[-1,1]$. Moreover, $n-1$ zeros of $\hat{B}_{n}(x)$ lie on $(-1,1)$ and the other one tends to $c$.

ii)

$$
\lim _{n \rightarrow \infty} \frac{\hat{B}_{n}(x)}{\varphi(x)^{n}}=\left(\frac{\varphi^{-}(c)}{2}\right)^{1 / 2}\left(1-\frac{\varphi(c)}{\varphi(x)}\right) S(x)
$$

where $S(x)$ is the Szegö function of $(c-x) \mu^{\prime}(x)$, and the convergence is uniform on compact sets of $C \backslash[-1,1]$.

For $M=0$,

iii)

$$
\lim _{n \rightarrow \infty} \frac{\hat{B}_{n}(x)}{\tilde{q}_{n}(x)}=\frac{1}{2}\left(1-\frac{\varphi(c)}{\varphi(x)}\right)\left(1-\frac{\varphi^{-}(c)}{\varphi(x)}\right)
$$

uniformly on compact sets of $C \backslash[-1,1] . n-2$ zeros of $\hat{B}_{n}(x)$ lie on $(-1,1)$ one more tends to $c$ and the other tends to $[-1,1]$.

iv)

$$
\lim _{n \rightarrow \infty} \frac{\hat{B}_{n}(x)}{\varphi^{n}(x)}=\frac{1}{2}\left(1-\frac{\varphi(c)}{\varphi(x)}\right)\left(1-\frac{\varphi^{-}(c)}{\varphi(x)}\right) S(x)
$$

where $S(x)$ is the Szegö function of $(c-x)^{2} \mu^{\prime}(x)$, and the convergence is uniform on compact sets of $C \backslash[-1,1]$.

The following is also straightforward from the theorem, Corollary 2.3 i) When $N=0$,

$$
x \hat{B}_{n}(x)=\alpha_{n} \hat{B}_{n+1}(x)+\beta_{n} \hat{B}_{n}(x)+\alpha_{n-1} \hat{B}_{n-1}(x)
$$

and $\lim _{n \rightarrow \infty} \alpha_{n}=\frac{1}{2}, \quad \lim _{n \rightarrow \infty} \beta_{n}=0$.

ii) When $M=0$,

$$
\begin{aligned}
(x-c)^{2} \hat{B}_{n}(x)= & \alpha_{n} \hat{B}_{n+2}(x)+\beta_{n} \hat{B}_{n+1}(x)+\gamma_{n} \hat{B}_{n}(x) \\
& +\beta_{n-1} \hat{B}_{n-1}(x)+\alpha_{n-2} \hat{B}_{n-2}(x)
\end{aligned}
$$


and $\lim _{n \rightarrow \infty} \alpha_{n}=\frac{1}{4}, \lim _{n \rightarrow \infty} \beta_{n}=-c, \lim _{n \rightarrow \infty} \gamma_{n}=c^{2}+\frac{1}{2}$

In both cases we get

COROLLARY 2.4 If $f(x)$ is a continuous function in $[-1,1]$ then

$$
\lim _{n \rightarrow \infty} \int_{-1}^{1} f(x) \hat{B}_{n}(x) \hat{B}_{n+k}(x) d \mu(x)=\frac{1}{\pi} \int_{-1}^{1} f(x) T_{k}(x) \frac{d x}{\sqrt{1-x^{2}}}
$$

Finally, we include here the maximality of the polynomials in the Sobolev space for the different possible choices of $M$ and $N$.

THEOREM 2.5 i) The family $\phi=\left\{(x-c)^{2 n}\right\}_{n=1}^{\infty}$ is maximal in the space $L^{2}(\mu)$.

ii) $\phi \cup\{1\}$ is maximal in $L^{2}(\mu+M \delta(x-c))$.

iii) When $N>0$, the family $\phi \cup\{1, x-c\}$ is maximal in the Hilbert space associated with the Sobolev product given in (1).

Proof If $\int_{-1}^{1}(x-c)^{2 n} f(x) d \mu(x)=0$ for $n=1,2, \ldots$ and for a function $f \in L^{2}(\mu) \cup L^{2}(\mu+M \delta(x-c))$ or in the Sobolev space, one has

$$
\begin{aligned}
0 & =\int_{\left[(c-1)^{2},(c+1)^{2}\right]} t^{n} f(c+\sqrt{t}) d \mu(c+\sqrt{t}) \\
& =\int_{\left[(c-1)^{2},(c+1)^{2}\right]} t^{n} f(c+\sqrt{t}) d v(t)
\end{aligned}
$$

which means that $t f(c+\sqrt{t})=0 v-a . e$. on $\left[(c-1)^{2},(c+1)^{2}\right]$. Thus $(x-c)^{2} f(x)=0 \mu-a . e$. on $[-1,1]$ and $f(x)=0 \mu$-a.e. also holds. So we have i).

If, moreover, $0=\int_{-1}^{1} f(x) d \mu(x)+M f(c)$, then $M f(c)=0$ and ii) follows. Finally, when $0=\langle f(x),(x-c)\rangle=\int_{-1}^{1} f(x)(x-c) d \mu(x)+N f^{\prime}(c)$ also holds, then $f^{\prime}(c)=0$ and we have iii)

We need a Christoffel-Darboux type formula which was proved in [3]. 
THEOREM 2.6 When $N>0$, the Sobolev polynomials $\hat{B}_{n}(x)$ satisfy the following Christoffel-Darboux type formula

$$
\begin{aligned}
{\left[(x-c)^{2}\right.} & \left.-(y-c)^{2}\right] \sum_{j=0}^{n} \hat{B}_{j}(x) \hat{B}_{j}(y) \\
= & \alpha_{n}\left[\hat{B}_{n+2}(x) \hat{B}_{n}(y)-\hat{B}_{n}(x) \hat{B}_{n+2}(y)\right]+\alpha_{n-1}\left[\hat{B}_{n+1}(x) \hat{B}_{n-1}(y)\right. \\
& \left.\quad-\hat{B}_{n-1}(x) \hat{B}_{n+1}(y)\right] \\
& +\beta_{n}\left[\hat{B}_{n+1}(x) \hat{B}_{n}(y)-\hat{B}_{n}(x) \hat{B}_{n+1}(y)\right]
\end{aligned}
$$

where $\alpha_{n}$ and $\beta_{n}$ are the coefficients of the five term recurrence relation of $\hat{B}_{n}(x)$

In case $N=0$, we have standard orthogonality as well as the standard Christoffel-Darboux formula.

\section{ESTIMATES FOR SOBOLEV POLYNOMIALS}

Because of

$$
\int_{-1}^{1} \tilde{q}_{n}(x) p_{j}(x) d \mu(x)=p_{j}(c) \int_{-1}^{1} \tilde{q}_{n}(x) d \mu(x), \quad j=0,1, \ldots, n,
$$

one can write

$$
\tilde{q}_{n}(x)=\lambda_{n} \sum_{k=0}^{n} p_{k}(c) p_{k}(x)=\lambda_{n} x_{n} \frac{p_{n+1}(x) p_{n}(c)-p_{n+1}(c) p_{n}(x)}{x-c}
$$

where $\alpha_{n}$ are the coefficients of the recurrence relation of $p_{n}(x)$ and $\hat{\lambda}_{n}=\int_{-1}^{1} \tilde{q}_{n}(x) d \mu(x)$. If $\mu$ belongs to the Szegö class, for every $x \in[-1,1]$,

$$
\begin{aligned}
\left|\tilde{q}_{n}(x)\right| & \leq \frac{\left|\lambda_{n} \alpha_{n} p_{n}(c)\right|}{|x-c|}\left(\left|p_{n+1}(x)\right|+\frac{\left|p_{n+1}(c)\right|}{\left|p_{n}(c)\right|}\left|p_{n}(x)\right|\right) \\
& \leq K_{1}\left(\left|p_{n+1}(x)\right|+\left|p_{n}(x)\right|\right)
\end{aligned}
$$


for some constant $K_{1}$ and for $n$ large enough, because

$$
\begin{aligned}
\lambda_{n} p_{n}(c) & =\int_{-1}^{1} \tilde{q}_{n}(x) p_{n}(c) d \mu(x) \\
& =\int_{-1}^{1} \tilde{q}_{n}(x)\left(p_{n}(c)+(x-c) \sum_{k=1}^{n} p_{n}^{(k)}(c)(x-c)^{k-1}\right) d \mu(x) \\
& =\int_{-1}^{1} \tilde{q}_{n}(x) p_{n}(x) d \mu(x)=\frac{k\left(\tilde{q}_{n}\right)}{k\left(p_{n}\right)}
\end{aligned}
$$

and $k\left(\tilde{q}_{n}\right) / k\left(p_{n}\right), p_{n+1}(c) / p_{n}(c)$ and $\alpha_{n}$ have limit points. So we have

COROLlaRY 3.1 Let $\mu$ be a measure on $[-1,1]$ which belongs to the Szegö class. Then, for every $x \in[-1,1]$,

$$
\left|\tilde{q}_{n}(x)\right| \leq K_{1}\left(\left|p_{n+1}(x)\right|+\left|p_{n}(x)\right|\right)
$$

for a constant $K_{1}$ which does not depend on $x$ and for $n$ large enough.

Taking into account that $\left(q_{n}(x)\right)_{n=0}^{\infty}$ are the orthonormal polynomials with respect to $(c-x)(c-x) d \mu(x)$, writting $q_{n}(x)$ in terms of $\tilde{q}_{k}(x)$, in the same way as before we have

CoRollary 3.2 If $\mu$ belongs to the Szegö class then for every $x \in[-1,1]$,

$$
\left|q_{n}(x)\right| \leq K_{2}\left(\left|p_{n+2}(x)\right|+\left|p_{n+1}(x)\right|+\left|p_{n}(x)\right|\right)
$$

for $n$ large enough and for some positive real constant $K_{2}$ which does not depend on $x$.

Using now Theorems 2.1 and 2.4, if $N>0$ then

$$
\hat{B}_{n}(x)=A_{n} q_{n}(x)+B_{n} q_{n-1}(x)+C_{n} q_{n-2}(x)
$$

where $A_{n}, B_{n}, C_{n}$ have limit points. In case $N=0$ then

$$
\hat{B}_{n}(x)=A_{n} \tilde{q}_{n}(x)+B_{n} \tilde{q}_{n-1}(x)
$$


from Theorem 2.4. Then

COROLlaRY 3.3 Let $\mu$ be a measure in the Szegö class. Then, for every $x \in[-1,1]$,

i) when $N>0$,

$$
\left|\hat{B}_{n}(x)\right| \leq K_{3}\left(\left|p_{n+3}(x)\right|+\left|p_{n+2}(x)\right|+\left|p_{n+1}(x)\right|+\left|p_{n}(x)\right|+\left|p_{n-1}(x)\right|\right)
$$

for $n$ large enough and for some positive real constant $K_{3}$ independent of $x$.

ii) when $N=0$ there is a constant $K_{3}^{*}$ such that

$$
\left|\hat{B}_{n}(x)\right| \leq K_{3}^{*}\left(\left|p_{n+1}(x)\right|+\left|p_{n}(x)\right|+\left|p_{n-1}(x)\right|\right)
$$

for $n$ large enough.

COROLLARY 3.4 If $\mu$ belongs to the Szegö class and there is a function $h(x)$ such that the orthonormal polynomials $p_{n}(x)$ satisfy $\left|p_{n}(x)\right| \leq h(x)$, $x \in[-1,1]$, then there is a constant $K$ such that

$$
\left|\hat{B}_{n}(x)\right| \leq K h(x)
$$

for $n$ large enough and for every $x \in[-1,1]$.

In particular, if $\mu$ is the Jacobi measure we know the function $h(x)$ and it will be very useful for the study of Fourier series.

Also in order to study Fourier series of Sobolev polynomials, we need estimates for $\hat{B}_{n}(c)$ and $\hat{B}_{n}^{\prime}(c)$.

For $M>0$, one has $0=\left\langle\hat{B}_{n}, 1\right\rangle=\int_{-1}^{1} \hat{B}_{n}(x) d \mu(x)+M \hat{B}_{n}(c)$, so

$$
\left|\hat{B}_{n}(c)\right|=\frac{1}{M}\left|\int_{-1}^{1} \hat{B}_{n}(x) d \mu(x)\right|
$$

In the same way, when $N>0$,

$$
\left|\hat{B}_{n}^{\prime}(c)\right|=\frac{1}{N}\left|\int_{-1}^{1}(c-x) \hat{B}_{n}(x) d \mu(x)\right|
$$


Taking into account that

$$
\hat{B}_{n}(x)=A_{n} q_{n}(x)+B_{n} q_{n-1}(x)+C_{n} q_{n-2}(x)
$$

where, as before, $q_{n}(x)$ are the orthonormal polynomials with respect to $(x-c)^{2} d \mu(x)$, in order to estimate $\hat{B}_{n}(c)$ and $\hat{B}_{n}^{\prime}(c)$ we only have to estimate $\int_{-1}^{1} q_{n}(x) d \mu(x)$, and $\int_{-1}^{l} q_{n}(x)(x-c) d \mu(x)$ respectively.

Moreover

$$
\begin{array}{r}
\int_{-1}^{1} q_{n}(x)(x-c) d \mu(x)=\frac{1}{q_{n}(c)} \int_{-1}^{1} q_{n}(x) q_{n}(c)(x-c) d \mu(x) \\
=\frac{1}{q_{n}(c)} \int_{-1}^{1} q_{n}(x)\left(q_{n}(c)+(x-c) \pi_{n-1}(x)\right)(x-c) d \mu(x)
\end{array}
$$

for any polynomial $\pi_{n-1}(x)$ of degree at most $n-1$. Then

$$
\int_{-1}^{1} q_{n}(x)(x-c) d \mu(x)=\frac{1}{q_{n}(c)} \int_{-1}^{1}(x-c) q_{n}^{2}(x) d \mu(x)
$$

Analogously,

$$
\begin{aligned}
\int_{-1}^{1} q_{n}(x) d \mu(x)= & \frac{1}{q_{n}(c)} \int_{-1}^{1} q_{n}(x)\left(q_{n}(c)+q_{n}^{\prime}(c)(x-c)\right) d \mu(x) \\
& -\frac{q_{n}^{\prime}(c)}{q_{n}(c)} \int_{-1}^{1} q_{n}(x)(x-c) d \mu(x) \\
= & \frac{1}{q_{n}(c)} \int_{-1}^{1} q_{n}^{2}(x) d \mu(x)+\frac{q_{n}^{\prime}(c)}{q_{n}^{2}(c)} \int_{-1}^{1} q_{n}^{2}(x)(c-x) d \mu(x) .
\end{aligned}
$$

Then, for a measure in Szegö class, we get

$$
\begin{aligned}
\int_{-1}^{1} q_{n}(x)(x-c) d \mu(x) & =O\left(\left(c-\sqrt{c^{2}-1}\right)^{n}\right), \int_{-1}^{1} q_{n}(x) d \mu(x) \\
& =O\left(n\left(c-\sqrt{c^{2}-1}\right)^{n}\right) .
\end{aligned}
$$


So, we have

THEOREM 3.1 If $\mu$ is a measure on $[-1,1]$ which belongs to the Szegö class and $M>0, N>0$, then there are constants $K_{1}$ and $K_{2}$ such that i) $\left|\hat{B}_{n}(c)\right| \leq K_{1} n r_{o}^{n}$

ii) $\left|\hat{B}_{n}^{\prime}(c)\right| \leq K_{2} r_{0}^{n}$

where $0<r_{0}=c-\sqrt{c^{2}-1}<1$

In the same way we get

THEOREM 3.2 Let $\mu$ be a measure in Szegö class.

i) When $N=0$, there is a constant $K$ such that $\left|\hat{B}_{n}(c)\right| \leq K r_{0}^{n}$.

ii) When $M=0$, there is a constant $K$ such that $\left|\hat{B}_{n}^{\prime}(c)\right| \leq K r_{0}^{n}$ where $r_{0}=c-\sqrt{c^{2}-1}<1$

\section{FOURIER SERIES}

Since $L^{2}(\mu)$ is a Hilbert space, it is clear that the space $S$ given by

$$
S=\left\{f(x): \int_{-1}^{1}|f(x)|^{2} d \mu(x)<\infty, \text { and } f^{\prime}(c) \text { exists }\right\}
$$

with the associated norm $\|\cdot\|_{s}$ derived from the Sobolev inner product

$$
\langle f, g\rangle=\int_{-1}^{l} f(x) g(x) d \mu(x)+M f(c) g(c)+N f^{\prime}(c) g^{\prime}(c)
$$

is also a Hilbert space because $\|f(x)\|_{s}^{2}=\|f(x)\|_{\mu}^{2}+M f^{2}(c)+N\left(f^{\prime}(c)^{2}\right)$ and a Cauchy sequence in $S$ is a Cauchy sequence in $L^{2}(\mu)$ and in the point $c$. Moreover, the maximality of the polynomials was seen in Theorem 2.5. So, $S_{n}(x ; f) \rightarrow f(x)$ in $S$ for any function of $S$, where

$$
S_{n}(x ; f)=\sum_{j=0}^{n}\left\langle\hat{B}_{j}(t), f(t)\right\rangle \hat{B}_{j}(x)
$$

is the partial sum of the Fourier-Sobolev series of $f$. In particular, it means that

$$
\lim _{n \rightarrow \infty} S_{n}(c ; f)=f(c), \lim _{n \rightarrow \infty} S_{n}^{\prime}(c ; f)=f^{\prime}(c)
$$


If we apply this to the functions $f_{1}(x)$ and $f_{2}(x)$ defined by

$$
\begin{aligned}
& f_{1}(x)=0 \text { if } x \in|-1,1|, f_{1}(c)=1, f_{1}^{\prime}(c)=0, \\
& f_{2}(x)=0 \text { if } x \in|-1,1|, f_{2}(c)=0, f_{2}^{\prime}(c)=1,
\end{aligned}
$$

their Fourier-Sobolev series are $M \sum_{k=0}^{\infty} \hat{B}_{k}(c) \hat{B}_{k}(x)$ and $N \sum_{k=0}^{\infty} \hat{B}_{k}^{\prime}(c) \hat{B}_{k}(x)$ respectively. Thus, we get

$$
\begin{aligned}
& M \sum_{k=0}^{n} \hat{B}_{k}(c) \hat{B}_{k}(x) \rightarrow f_{1}(x) \text { in } S \\
& M \sum_{k=0}^{n} \hat{B}_{k}(c) \hat{B}_{k}(x) \rightarrow f_{2}(x) \text { in } S
\end{aligned}
$$

which means that

$$
\begin{gathered}
c 1: \sum_{k=0}^{n} \hat{B}_{k}(c) \hat{B}_{k}(x) \rightarrow 0 \text { in } L_{2}(\mu), \\
c 2: \sum_{k=0}^{n} \hat{B}_{k}^{\prime}(c) \hat{B}_{k}(x) \rightarrow 0 \text { in } L_{2}(\mu), \\
c 3: \sum_{k=0}^{\infty}\left(\hat{B}_{k}(c)\right)^{2}=1 / M, \\
c 4: \sum_{k=0}^{\infty}\left(\hat{B}_{k}^{\prime}(c)\right)^{2}=1 / N, \\
c 5: \sum_{k=0}^{\infty} \hat{B}_{k}(c) \hat{B}_{k}^{\prime}(c)=0 .
\end{gathered}
$$

From now on, the Jacobi measure, $d \mu(x)=(1-x)^{\alpha}(1+x)^{\beta} d x, \alpha>-1$, $\beta>-1$, will be considered, and the behaviour of the corresponding Fourier-Sobolev series will be studied.

We know that the Jacobi orthonormal polynomials $p_{n}^{(\alpha, \beta)}(x)$ satisfy (see [7], Theorem 3.14 in page 101)

$$
(1-x)^{\frac{\alpha}{2}+\frac{1}{4}}(1+x)^{\frac{\beta}{2}+\frac{1}{4}}\left|p_{n}^{(x, \beta)}(x)\right| \leq C \quad x \in[-1,1] .
$$


Then, for the corresponding Sobolev orthonormal polynomials $\hat{B}_{n}^{(\alpha, \beta)}(x)$, Corollary 3.4 yields the uniform bound

$$
\left|\hat{B}_{n}^{(x, \beta)}(x)\right| \leq \frac{K}{(1-x)^{\frac{\alpha}{2}+\frac{1}{4}}(1+x)^{\frac{\beta}{2}+\frac{1}{4}}}=h^{(x, \beta)}(x) \quad \text { for } x \in(-1,1)
$$

for some constant $K$ and for $n$ large enough (we will continue denoting by $\hat{B}_{n}(x)$ the polynomials $\hat{B}_{n}^{(\alpha, \beta)}(x)$ ). From Theorem 3.1 and 3.2 , for $k$ large enough, every term of the series $\sum_{k=0}^{\infty} \hat{B}_{k}(c) \hat{B}_{k}(x)$ has the majorant $K^{*} k\left(c-\sqrt{c^{2}-1}\right)^{k}$ for some constant $K^{*}$ in closed subsets of $(-1,1)$. Then $\sum_{k=0}^{\infty} \hat{B}_{k}(c) \hat{B}_{k}(x)$ converges for $x \in(-1,1)$ and uniformly in any compact set $[-1+c, 1-c], 0<c<1$. Hence, $\sum_{k=0}^{\infty} \hat{B}_{k}(c) \hat{B}_{k}(x)$ is a continuous function for $x \in(-1,1)$ which, from condition $\mathrm{cl}$, equals zero $\mu$-a.e. in $[-1,1]$ provided that $M>0$. As a consequence, $\sum_{k=0}^{\infty} \hat{B}_{k}(c) \hat{B}_{k}(x)=0, x \in(-1,1)$. In the same way, Theorems 3.1 and 3.2 and condition $c 2$ give $\sum_{k=0}^{\infty} \hat{B}_{k}^{\prime}(c) \hat{B}_{k}(x)=0, x \in(-1,1)$ provided that $N>0$.

THEOREM 4.1 Let $\hat{B}_{n}(x)$ be the orthonormal polynomials with respect to the Sobolev inner product associated with the Jacobi measure. Then

i) When $M>0, \sum_{k=0}^{\infty} \hat{B}_{k}(c) \hat{B}_{k}(x)=0$ for every $x \in(-1,1)$.

ii) When $N>0, \sum_{k=0}^{\infty} \hat{B}_{k}^{\prime}(c) \hat{B}_{k}(x)=0$ for every $x \in(-1,1)$

Now, we can prove the pointwise convergence of $S_{n}(x ; f)$ to $f(x)$ when one has standard sufficient conditions for the function $f(x)$.

THEOREM 4.2 Let $x_{0} \in(-1,1)$ and let $f$ be a function with derivative in $c$ such that $\left(f\left(x_{0}\right)-f(t)\right) /\left(x_{0}-t\right) \in L^{2}(\mu)$ where $\mu$ is the Jacobi measure. Then

$$
\begin{aligned}
& \text { i) } \sum_{k=0}^{\infty} c_{k} \hat{B}_{k}\left(x_{0}\right)=f\left(x_{0}\right), \\
& \text { ii) } \sum_{k=0}^{\infty} c_{k} \hat{B}_{k}(c)=f(c) \text { if } M>0, \\
& \text { iii) } \sum_{k=0}^{\infty} c_{k} \hat{B}_{k}^{\prime}(c)=f^{\prime}(c) \text { if } N>0
\end{aligned}
$$

where $c_{k}=\left\langle f, \hat{B}_{k}\right\rangle$. 
Proof Because of $f \in L^{2}(\mu)$ when $\left(f\left(x_{0}-f(t)\right) /\left(x_{0}-t\right) \in L^{2}(\mu)\right.$, ii) and iii) are proved, so we only have to prove i). Let us denote $D_{n}(x, t)=\sum_{k=0}^{n} \hat{B}_{k}(x) \hat{B}_{k}(t)$. Since

$$
\begin{aligned}
f\left(x_{0}\right)-S_{n}\left(x_{0} ; f\right)= & \left\langle f\left(x_{0}\right)-f(t), D_{n}\left(x_{0}, t\right)\right\rangle \\
= & \int_{-1}^{1}\left(f\left(x_{0}\right)-f(t)\right) D_{n}\left(x_{0}, t\right) d \mu(t)+M\left(f\left(x_{0}\right)\right. \\
& -f(c)) D_{n}\left(x_{0}, c\right)-\left.f^{\prime}(c) \frac{\partial D_{n}}{\partial t}\left(t, x_{0}\right)\right|_{t=c},
\end{aligned}
$$

Theorem 4.1 yields

$$
\left.\lim _{n \rightarrow \infty}\left(f\left(x_{0}\right)-S_{n}\left(x_{0} ; f\right)\right)=\lim _{n \rightarrow \infty} \int_{-1}^{1}\left(f\left(x_{0}\right)-f(t)\right) D_{n}\left(x_{0}, t\right)\right) d \mu(t)
$$

On the other hand, Christoffel-Darboux formula gives

$$
\begin{aligned}
& \left|\int_{-1}^{1}\left(f\left(x_{0}\right)-f(t)\right) D_{n}\left(x_{0}, t\right) d \mu(t)\right| \\
& \quad \leq \alpha_{n}\left|\hat{B}_{n+2}\left(x_{0}\right)\right|\left|\int_{-1}^{1} \frac{f\left(x_{0}\right)-f(t)}{\left(x_{0}-c\right)^{2}-(t-c)^{2}} \hat{B}_{n}(t) d \mu(t)\right| \\
& \quad+\alpha_{n}\left|\hat{B}_{n}\left(x_{0}\right)\right| \\
& \left.\quad \cdot \mid \int_{-1}^{1} \frac{f\left(x_{0}\right)-f(t)}{\left(x_{0}-c\right)^{2}-(t-c)^{2}} \hat{B}_{n+2}(t)\right) d \mu(t)\left|+\alpha_{n-1}\right| \hat{B}_{n+1}\left(x_{0}\right) \mid \\
& \left.\quad \cdot \mid \int_{-1}^{1} \frac{f\left(x_{0}\right)-f(t)}{\left(x_{0}-c\right)^{2}-(t-c)^{2}} \hat{B}_{n-1}(t)\right) d \mu(t)\left|+\alpha_{n-1}\right| \hat{B}_{n-1}\left(x_{0}\right) \mid \\
& \quad \cdot\left|\int_{-1}^{1} \frac{f\left(x_{0}\right)-f(t)}{\left(x_{0}-c\right)^{2}-(t-c)^{2}} \hat{B}_{n+1}(t) d \mu(t)\right|+\left|\beta_{n}\right|\left|\hat{B}_{n+1}\left(x_{0}\right)\right| \\
& \quad \cdot\left|\int_{-1}^{1} \frac{f\left(x_{0}\right)-f(t)}{\left(x_{0}-c\right)^{2}-(t-c)^{2}} \hat{B}_{n}(t) d \mu(t)\right|+\left|\beta_{n}\right| \\
& \left.\quad \cdot \mid \hat{B}_{n}\left(x_{0}\right) \int_{-1}^{1} \frac{f\left(x_{0}\right)-f(t)}{\left(x_{0}-c\right)^{2}-(t-c)^{2}} \hat{B}_{n+1}(t)\right) d \mu(t) \mid
\end{aligned}
$$


Furthermore, $\alpha_{n}$ and $\beta_{n}$ are bounded according to Theorem 2.2 and Corollary 2.3. $\left|\hat{B}_{n}\left(x_{0}\right)\right| \leq h^{(\alpha, \beta)}\left(x_{0}\right)<\infty$ also holds, and since $\left|\left(x_{0}-c\right)^{2}-(t-c)^{2}\right| \geq 2\left|x_{0}-t\right|(c-1)$ when $x_{0}, \quad t \in[-1,1]$, the function $f\left(x_{0}\right)-f(t) /\left(x_{0}-c\right)^{2}-(t-c)^{2}$ belongs to $L^{2}(\mu)$ when $f\left(x_{0}\right)-f(t) / x_{0}-t \in L^{2}(\mu)$. Hence, it also belongs to $L^{2}\left((t-c)^{2} d \mu(t)\right)$ and

$$
\lim _{n \rightarrow \infty} \int_{-1}^{1} \frac{f\left(x_{0}\right)-f(t)}{\left(x_{0}-c\right)^{2}-(t-c)^{2}} \hat{B}_{n}(t) d \mu(t)=0
$$

follows from Theorems 2.1 and 2.4. As a consequence, each term in the last sum tends to zero and the theorem is proved.

THEOREM 4.3 Let $f(x)$ be a function with first derivative in $c$ satisfying a Lipschitz condition of order $\eta<1$ uniformly in $[-1,1]$, i.e. $|f(x+h)-f(x)| \leq K|h|^{\eta}$ for $|h|<\delta$ for some $\delta>0$. If $c_{k}=\left\langle f, \hat{B}_{k}\right\rangle$, then

$$
\lim _{n \rightarrow \infty} \sum_{k=0}^{\infty} c_{k} \hat{B}_{k}(x)=f(x), \quad x \in(-1,1)
$$

and the convergence is uniform in $[-1+c, 1-c]$ for every $c$ such that $0<c<1$. Moreover $\sum_{k=0}^{\infty} c_{k} \hat{B}_{k}(c)=f(c)$ when $M>0$ and $\sum_{k=0}^{\infty} c_{k} \hat{B}_{k}^{\prime}(c)=f^{\prime}(c)$ if $N>0$.

Proof In the same way as before, we only have to prove that $\int_{-1}^{1} f(t) D_{n}(x, t) d \mu(t)$ converges to $f(x)$. Moreover,

$$
\begin{aligned}
\left|f(x)-\int_{-1}^{1} f(t) D_{n}(x, t) d \mu(t)\right|= & \left|\int_{-1}^{1}(f(x)-f(t)) D_{n}(x, t) d \mu(t)\right| \\
\leq & \left|\int_{|x-t| \geq \delta}(f(x)-f(t)) D_{n}(x, t) d \mu(t)\right| \\
& +\left|\int_{|x-t|<\delta}(f(x)-f(t)) D_{n}(x, t) d \mu(t)\right| \\
= & I_{n}^{(1)}(x)+I_{n}^{(2)}(x) .
\end{aligned}
$$


Since $\quad f(x)-f(t) /(x-c)^{2}-(t-c)^{2}\left(1-\chi_{(x-\delta, x+\delta)}(t)\right), \quad$ where $\chi_{(x-\delta, x+\delta)}(t)$ is the characteristic function of the interval, belongs to $L^{2}(\mu)$, using the Christoffel-Darboux formula and with the same procedure as in the last Theorem, the term $I_{n}^{(1)}(x)$ tends to zero uniformly in closed subintervals of $(-1,1)$. For $I_{n}^{2}(x)$ we can write

$$
\begin{aligned}
I_{n}^{(2)}(x) \leq & \alpha_{n}\left|\hat{B}_{n+2}(x)\right|\left|\int_{|x-t|<\delta} \frac{f(x)-f(t)}{(x-c)^{2}-(t-c)^{2}} \hat{B}_{n}(t) d \mu(t)\right| \\
& \left.+\alpha_{n}\left|\hat{B}_{n}(x)\right| \mid \int_{|x-t|<\delta} \frac{f(x)-f(t)}{(x-c)^{2}-(t-c)^{2}} \hat{B}_{n+2}(t)\right) d \mu(t) \mid \\
& \left.+\alpha_{n-1}\left|\hat{B}_{n+1}(x)\right| \mid \int_{|x-t|<\delta} \frac{f(x)-f(t)}{(x-c)^{2}-(t-c)^{2}} \hat{B}_{n-1}(t)\right) d \mu(t) \mid \\
& \left.+\alpha_{n-1}\left|\hat{B}_{n-1}(x)\right| \mid \int_{|x-t|<\delta} \frac{f(x)-f(t)}{(x-c)^{2}-(t-c)^{2}} \hat{B}_{n+1}(t)\right) d \mu(t) \mid \\
& +\left|\beta_{n}\right|\left|\hat{B}_{n+1}(x)\right|\left|\int_{|x-t|<\delta} \frac{f(x)-f(t)}{(x-c)^{2}-(t-c)^{2}} \hat{B}_{n}(t) d \mu(t)\right| \\
& \left.+\left|\beta_{n}\right|\left|\hat{B}_{n}(x)\right| \mid \int_{|x-t|<\delta} \frac{f(x)-f(t)}{(x-c)^{2}-(t-c)^{2}} \hat{B}_{n+1}(t)\right) d \mu(t) \mid .
\end{aligned}
$$

Lipschitz condition gives

$$
\begin{aligned}
& \left|\int_{|x-t|<\delta} \frac{f(x)-f(t)}{(x-c)^{2}-(t-c)^{2}} \hat{B}_{n}(t) d \mu(t)\right| \\
& \quad \leq \int_{|x-t|<\delta} \frac{K\left|\hat{B}_{n}(t)\right|}{|x-t|^{1-\eta}|x+t-2 c|} d \mu(t) \\
& \quad \leq \frac{K\left(h^{(\alpha, \beta)}(x)+O(1)\right)}{(c-1)^{2}} \int_{|x-t|<\delta} \frac{d \mu(t)}{|x-t|^{1-\eta}}
\end{aligned}
$$

Hence,

$$
I_{n}^{(2)}=O\left(\int_{|x-t|<\delta} \frac{d \mu(t)}{|x-t|^{1-\eta}}\right)
$$

and, as a consequence, $\int_{-1}^{1}(f(x)-f(t)) D_{n}(x, t) d \mu(t)$ tends to zero uniformly in any closed subinterval of $(-1,1)$. 
Let us denote, as usual,

$$
\omega(\delta)=\omega(\delta, f)=\sup \left\{\left|f\left(x_{1}\right)-f\left(x_{2}\right)\right|: x_{1}, x_{2} \in[-1,1],\left|x_{1}-x_{2}\right| \leq \delta\right\}
$$

the modulus of continuity of a function $f(x)$ in $[-1,1]$.

THEOREM 4.4 Let $f(x)$ be a function such that its modulus of continuity $w(\delta)$ satisfies the condition

$$
\omega(\delta)=O\left(\log ^{-(1+\epsilon)} \frac{1}{\delta}\right)
$$

for $c>0$, and with first derivative in c. If $c_{k}=\left\langle f, \hat{B}_{k}\right\rangle$, $\sum_{k=0}^{\infty} c_{k} \hat{B}_{k}(x)=f(x)$ a.e. $\operatorname{in}[-1,1]$. Moreover, $\quad \sum_{k=0}^{\infty} c_{k} \hat{B}_{k}(c)=f(c)$ provided that $M>0$, and $\sum_{k=0}^{\infty} c_{k} \hat{B}_{k}^{\prime}(c)=f^{\prime}(c)$ when $N>0$.

Proof It is clear that the modulus of continuity of the function $f(x) /(x-c)^{2}$ satisfies the condition

$$
\omega\left(\delta, \frac{f(x)}{(x-c)^{2}}\right)=O\left(\log ^{-(1+c)} \frac{1}{\delta}\right)
$$

Let $d_{k}=\int_{-1}^{1} f(x) /(x-c)^{2} q_{k}(x)(x-c)^{2} d \mu(x)$. By Jackson's Approximation Theorem (see [7] Chap I), there is a polynomial $\pi_{n}(x)$ such that

$$
\left|\frac{f(x)}{(x-c)^{2}}-\pi_{n}(x)\right|=O\left(\frac{1}{\log ^{1+c} n}\right)
$$

whence

$$
\sum_{k=n}^{\infty} d_{k}^{2}=\int_{-1}^{1}\left(\frac{f(x)}{(x-c)^{2}}-\pi_{n}(x)\right)^{2}(x-c)^{2} d \mu(x)=O\left(\frac{1}{\log ^{2+2 \epsilon} n}\right)
$$

Taking into account that, from Theorems 2.1 and 2.4, $c_{k}=\left\langle f, \hat{B}_{k}\right\rangle=A_{k} d_{k}+B_{k} d_{k-1}+C_{k} d_{k-2}+M f(c) \hat{B}_{k}(c)+N f^{\prime}(c) \hat{B}_{k}^{\prime}(c)$, 
as well as $\sum_{k=n}^{\infty} d_{k} d_{k-1} \leq\left(\sum_{k=n}^{\infty} d_{k}^{2}\right)^{1 / 2}\left(\sum_{k=n}^{\infty} d_{k-1}^{2}\right)^{1 / 2}$ and the estimates for $\hat{B}_{k}(c)$ and for $\hat{B}_{k}^{\prime}(c)$, we get

$$
\sum_{k=n}^{\infty} c_{k}^{2}=O\left(\frac{1}{\log ^{2+2 \epsilon} n}\right)
$$

As a consequence (see Theorem 3.3 in pag. 137 of [7]), $\sum_{k=0}^{\infty} c_{k}^{2} \log ^{2} k<\infty$ and it yields (see Theorem 2.5 in pag. 126 of [7]), $\sum_{k=0}^{n} c_{k} \hat{B}_{k}(x)$ converges a.e. $x \in[-1,1]$ (here one has to take into account that $\int_{-1}^{1} g^{2}(x) d \mu(x) \leq\langle g, g\rangle$ for every function $g$ in $S$. But, since $f(x)$ is a continuous function, $\sum_{k=0}^{n} c_{k} \hat{B}_{k}(x)$ converges to $f(x)$ in the Sobolev space. Then

$$
\sum_{k=0}^{\infty} c_{k} \hat{B}_{k}(x)=f(x), \quad \text { a.e. } x \in[-1,1]
$$

as well as $\sum_{k=0}^{\infty} c_{k} \hat{B}_{k}(c)=f(c)$ and $\sum_{k=0}^{\infty} c_{k} \hat{B}_{k}^{\prime}(c)=f^{\prime}(c)$.

\section{Acknowledgements}

The work of F. Marcellán was supported by a grant of Dirección General de Investigación (Ministerio de Ciencia y Tecnología) of Spain BFM-20000206-C04-01, and INTAS project INTAS 2000-272

\section{References}

[1] Alfaro M., Marcellán F., Rezola M. L., Estimates for Jacobi-Sobolev type orthogonal polynomials. Applic. Analysis 67 (1997) 157-174

[2] López G., Marcellán F., Van Assche W. Relative asymptotics for polynomials orthogonal with respect to a discrete Sobolev inner product, Constr. Approx. 11 (1995) 107-137.

[3] Marcellán F., Pérez T. E., Piñar M. Regular Sobolev type orthogonal polynomials: the Bessel case, Rocky Mount. J. of Math., 25 (4) (1995) 1431-1457.

[4] Marcellán F., Van Assche W. Relative asymptotics for orthogonal polynomials with a Sobolev inner product, J. Approx. Theory 72 (1992) 192-209.

[5] Maté A., Nevai P., Totik V. Asymptotics for the leading coefficients of orthogonal polynomials, Constr. Approx. 1 (1985) 63-69.

[6] Maté A., Nevai P., Totik V. Strong and weak convergence of orthogonal polynomials on the unit circle, Amer. J. Math. 109 (1987) 239-282.

[7] Osilenker B. Fourier Series in Orthogonal Polynomials, World Scientific, Singapore 1999.

[8] Szegö G. Orthogonal Polynomials, Amer. Math. Soc. Colloq. Pub. 23 Amer. Math. Soc. Providence, RI, 1975 (4th edition). 